\title{
Magnetic resonance imaging features of dogs with incomplete recovery after acute, severe spinal cord injury
}

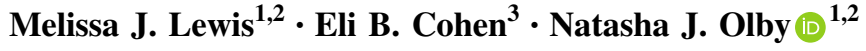 \\ Received: 27 May 2017 / Revised: 12 August 2017 / Accepted: 14 August 2017 / Published online: 23 October 2017 \\ (C) International Spinal Cord Society 2018
}

\begin{abstract}
Study design Retrospective case series.
\end{abstract}

Objectives Describe the magnetic resonance imaging (MRI) features of dogs chronically impaired after severe spinal cord injury (SCI) and investigate associations between imaging variables and residual motor function.

Setting United States of America.

Methods Thoracolumbar MRI from dogs with incomplete recovery months to years after clinically complete (paralysis with loss of pain perception) thoracolumbar SCI were reviewed. Lesion features were described and quantified. Gait was quantified using an ordinal, open field scale (OFS). Associations between imaging features and gait scores, duration of injury (DOI), or SCI treatment were determined.

Results Thirty-five dogs were included. Median OFS was 2 (0-6), median DOI was 13 months (3-83), and intervertebral disk herniation was the most common diagnosis $(n=27)$. Myelomalacia was the most common qualitative feature followed by cystic change; syringomyelia and fibrosis were uncommon. Lesion length corrected to L2 length (LL:L2) was variable (median LL:L2 = 3.5 (1.34-11.54)). Twenty-nine dogs had 100\% maximum cross-sectional spinal cord compromise (MSCC) at the lesion epicenter and the length of $100 \%$ compromised area varied widely (median length 100\% MSCC: L2 $=1.29(0.39-7.64))$. Length 100\% MSCC:L2 was associated with OFS $(p=0.012)$. OFS was not associated with any qualitative features. DOI or treatment type were not associated with imaging features or lesion quantification.

Conclusions Lesion characteristics on MRI in dogs with incomplete recovery after severe SCI were established. Length of $100 \%$ MSCC was associated with hind limb motor function. Findings demonstrate a spectrum of injury severity on MRI among severely affected dogs, which is related to functional status.

Electronic supplementary material The online version of this article (doi:10.1038/s41393-017-0004-8) contains supplementary material, which is available to authorized users

$\triangle$ Natasha J. Olby

njolby@ncsu.edu

1 Department of Clinical Sciences, College of Veterinary Medicine, North Carolina State University, Raleigh, NC, USA

2 Comparative Medicine Institute, North Carolina State University, Raleigh, NC, USA

3 Department of Molecular Biomedical Sciences, College of Veterinary Medicine, North Carolina State University, Raleigh, NC, USA

\section{Introduction}

Spinal cord injury (SCI) is common in dogs and magnetic resonance imaging (MRI) has become widespread in the diagnosis of acute canine SCI [1-7]. However, the appearance of severe injury on MRI in the chronic setting among dogs with an incomplete clinical recovery has not been well documented, and the relationship between specific imaging features in this population and functional status is unknown.

The MRI appearance of acute myelopathies in dogs has been documented with various qualitative and quantitative features [2, 3, 5, 6, 8-17]. Among these, the presence and dimensions of intramedullary hyperintensity on $\mathrm{T} 2 \mathrm{~W}$ images and length of cord compression have been associated with outcome for dogs suffering from acute intervertebral disk herniation $[6,10,13,18]$. The maximal cross-sectional 
area of intramedullary hyperintensity on $\mathrm{T} 2 \mathrm{~W}$ images has been associated with outcome in acute, non-compressive nucleus pulposus extrusion [3].

Regardless of functional outcome, dogs that suffered an acute, spontaneous SCI are not commonly imaged in the chronic phase unless there is an abrupt change in neurological status suggestive of a new injury. The spinal cord has been reported to be normal on MRI by 6-weeks post-injury for dogs that recover clinically following intervertebral disk herniation [19]. Dogs have been evaluated with MRI up to 16 weeks following experimental traumatic SCI and demonstrated MRI changes in the spinal cord (areas of relative hypointensity on $\mathrm{T} 1 \mathrm{~W}$ images and hyperintensity on $\mathrm{T} 2 \mathrm{~W}$ images) that mirrored histopathologically confirmed formation of a glial scar surrounding a fluid-filled cavitation at the lesion site [20]. Studies describing imaging features in the chronic setting among dogs left with permanent neurologic deficits after severe, spontaneous injury are lacking [21].

Specific MRI changes in acute and chronic SCI have been described in humans [22-27]. Abnormalities at the lesion site associated with worse neurologic recovery in acute cervical SCI in people include maximum spinal cord compression, hemorrhage, and spinal cord swelling [23]. Syrinx formation was more common in chronic patients when there was a functionally complete SCI [22]. More recently, structural atrophy distant to the site of injury, measured as the cross-sectional spinal cord area above the level of injury, has been associated with worse motor recovery after SCI [28-30].

Dogs with SCI share many overlapping features with human SCI, making them an attractive large animal model capable of capturing the heterogeneity (of both population and injury) that is lacking in experimental models [31]. The full spectrum of MRI features of dogs with chronic impairment after acute, complete injury needs to be described, and identification of imaging features that predict functional recovery would enhance their use in clinical trials on chronic paralysis. Our objective was to describe the MRI features of dogs with chronic SCI and to investigate associations between imaging variables and functional status. We hypothesized that smaller spinal cord lesion dimensions would be associated with greater motor function.

\section{Methods}

\section{Case selection}

The medical records of dogs that suffered an acute, severe thoracolumbar SCI in which a thoracolumbar MRI was performed at least 3 months after injury were reviewed. All dogs were participants in one or more clinical trials for chronic paralysis as part of the North Carolina State University College of Veterinary Medicine Canine Spinal Cord Injury Program. MRIs were performed as part of these studies, but prior to initiation of any therapy associated with the particular study. Prior therapies were noted but not considered exclusion criteria. To be included, the SCI needed to be severe enough to cause acute paralysis and loss of pain perception followed by incomplete recovery characterized by absent or minimal recovery of pain perception and chronic motor deficits. Data collected from the medical records included signalment, diagnosis, treatment(s) of the SCI, spinal cord lesion location, and duration of injury (interval from injury to chronic imaging). Treatments at the time of prior acute injury were broadly categorized as medical (consisting of any combination of supportive care, pain management, or rehabilitation) or surgical (consisting of spinal cord decompression with or without stabilization). Results of neurologic examination and gait status at the time of imaging were also obtained. Gait was quantified using an ordinal gait scale (open field score, ranging from 0 to 12) $[32,33]$. Open field score of $<4$ corresponds to taking no weight bearing steps, a score of 4 corresponds to taking weight bearing steps less than $10 \%$ of the time, and scores $\geq 5$ correspond to taking weight bearing steps between 10 and $100 \%$ of the time.

\section{Imaging acquisition}

All MRIs were performed using a $1.5 \mathrm{~T}$ scanner (Siemens Medical Solutions USA Inc., Malvern, PA) with a CP spine array coil to acquire standard transverse and sagittal sequences, including T1W pre-contrast and post-contrast, $\mathrm{T} 2 \mathrm{~W}$, short $\mathrm{T} 1$ inversion recovery, and half-Fourier acquisition single-shot turbo spin-echo (HASTTE) [34]. Additional sequences utilized in some, but not all, scans included proton density and gradient echo/T2* (Supplementary Information 1).

\section{Imaging analysis}

MRIs were reviewed for the presence or absence (Yes/No) of the following qualitative lesion features: extended spinal cord atrophy (associated with the injury site), myelomalacia, syrinx, cystic change, and intramedullary fibrosis (defined in Table 1) [27]. Gradient echo sequences were not commonly performed in this population; however, when available, they were evaluated along with the standard sequences for the presence of hemorrhage as an additional qualitative feature. The vertebral level of the lesion epicenter (lesion location) was noted. Dogs were also separated into a "cranial group" (lesion located T13-L1 interspace or cranial) and a "caudal group" (lesion located L1-L2 or 
Table 1 Qualitative imaging features

\begin{tabular}{|c|c|}
\hline Feature & Definition \\
\hline Extended atrophy & $\begin{array}{l}\text { Narrowing of the spinal cord in the mid-sagittal plane spanning at least two vertebral bodies in length; spinal cord } \\
\text { height subjectively compared to adjacent normal areas cranial and caudal to the lesion }\end{array}$ \\
\hline Myelomalacia & Ill-defined area of $\mathrm{T} 1$ hypointensity and moderate $\mathrm{T} 2$ hyperintensity (less intense than cerebrospinal fluid) \\
\hline Syringomyelia & $\begin{array}{l}\text { Well-defined region of T1 hypointensity, T2 and HASTE hyperintensity, isointense to cerebrospinal fluid extending } \\
>1 \text { vertebral body in length; tubular or loculated in shape }\end{array}$ \\
\hline Focal cystic change & As for syringomyelia but extending $<1$ vertebral body in length; round or oval in shape \\
\hline Intramedullary fibrosis & Area of $\mathrm{T} 1$ and $\mathrm{T} 2$ hypointensity on sagittal images \\
\hline Intramedullary hemorrhage & Area of $\mathrm{T} 1$ and $\mathrm{T} 2$ hypointensity with susceptibility artifact on $\mathrm{T} 2 * \mathrm{GRE}$ images \\
\hline
\end{tabular}

Table 2 Quantitative imaging variables

\begin{tabular}{ll}
\hline Feature & Definition \\
\hline Lesion length (LL) & $\begin{array}{l}\text { Length (in cm) of the abnormal spinal cord signal intensity visible on T2W sagittal } \\
\text { images with cranial and caudal extent corroborated on T2W transverse images; } \\
\text { also expressed as a ratio compared to the length of the L2 vertebral body } \\
\text { Area of abnormal spinal cord signal intensity at the lesion epicenter on T2W } \\
\text { transverse images expressed as a percentage of the total cross-sectional area of the } \\
\text { cord at that location }(0-100 \%)\end{array}$ \\
$\begin{array}{l}\text { Length (in cm) of the region with } 100 \% \text { MSCC on T2W sagittal images with the } \\
\text { cranial and caudal extent corroborated on T2W transverse images; also expressed } \\
\text { as a ratio compared to the length of the L2 vertebral body }\end{array}$
\end{tabular}

caudal) to evaluate the impact of lesion location on functional status. This cut-off was chosen since injury affecting the lumbar segments might disrupt intraspinal motor circuitry important for gait generation (the central pattern generator, CPG). Calculations characterizing the dimensions of the lesion were performed manually using commercially available software (Horos version 1.0.6) as defined in Table 2 [2]. Lesion length and length of the region within the lesion with $100 \%$ cross-sectional abnormal signal intensity were recorded as raw values and normalized to the length of vertebral body L2 to adjust for dogs of different sizes [2].

\section{Statistical analysis}

All analyses were performed using Jmp 12 Pro (SAS Institute, Cary, NC, USA). Qualitative features were treated as categorical data and reported as frequency of occurrence. Summary statistics for continuous data (quantitative variables) were reported as mean and standard deviation if normally distributed, or median and interquartile range (IQR) if not using the Wilk-Shapiro test for normality. The effect of the presence of specific qualitative features or lesion location on open field score as well as the relationship between duration of injury and gait scores were determined using a Wilcoxon rank sum test. Linear regression and a one-way ANOVA were used to compare quantitative variables and open field score or duration of injury. The effect of type of treatment (medical vs. surgical) on the presence of qualitative features were established by constructing contingency tables and using a Fisher's exact test, whereas the effect of type of treatment on quantitative variables was determined using a Wilcoxon rank sum test. $p<0.05$ significant with adjusted $p$-values calculated for multiple comparisons using Holm's correction calculator.

We certify that all applicable institutional and governmental regulations concerning the ethical use of animals were followed during the course of this research (protocol numbers D11-015-O, 15-004-01).

\section{Results}

Thirty-five dogs met the inclusion criteria (Supplementary Information 1). There were 13 Dachshunds, 8 mixed breed dogs, 3 Pit Bull Terriers, 2 each of miniature schnauzers, Australian cattle dogs, Shih Tzus and French Bulldogs, and 1 each of Cocker Spaniel, Boston Terrier, and Bichon Frise. Mean age was 5.5 years (SD 2.24) and median body weight was $8.8 \mathrm{~kg}$ (IQR: 6.5-14.6). Intervertebral disk herniation was the most common diagnosis $(n=27)$, followed by fibrocartilaginous embolism $(n=2)$, vertebral column fracture $(n=5)$, and traumatic intervertebral disk extrusion $(n=1)$. For dogs with intervertebral disk herniation, 19 were treated with decompressive surgery at the time of injury and 8 were managed conservatively. Surgery was 

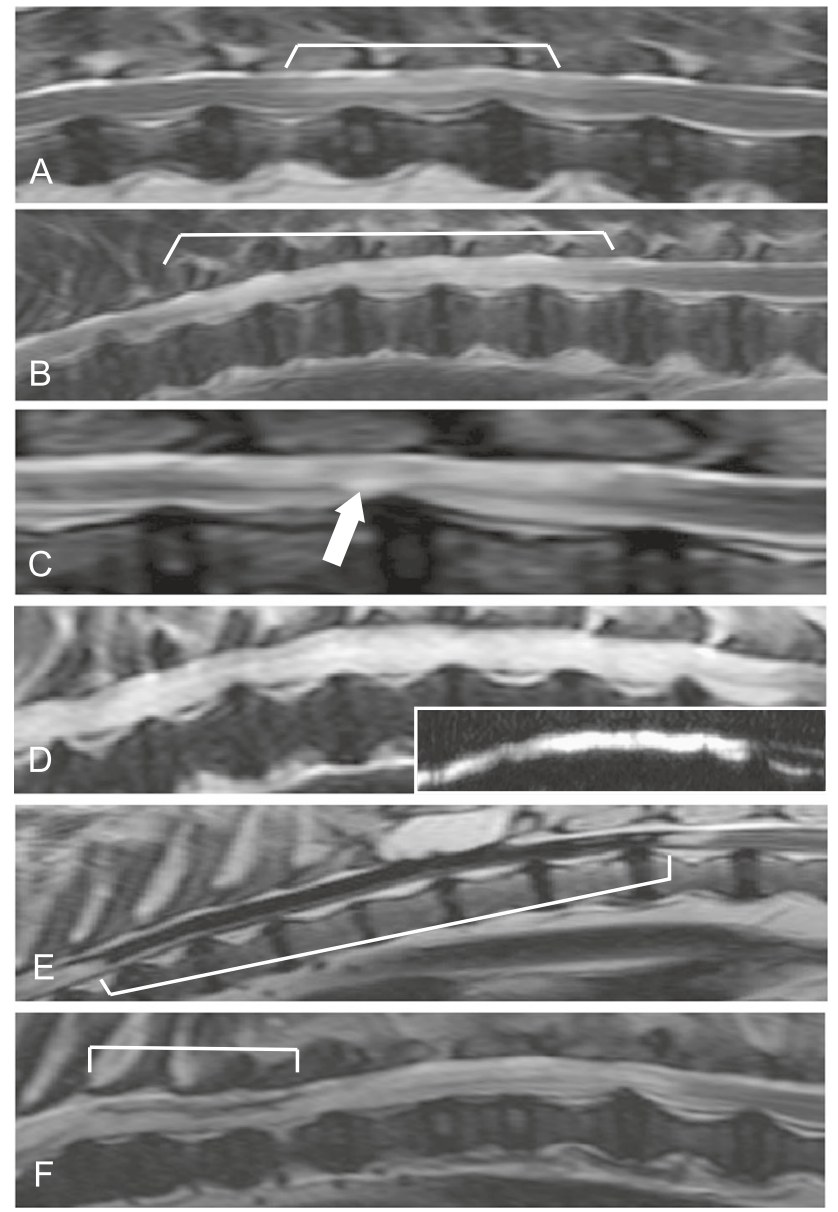

Fig. 1 Representative images depicting qualitative imaging abnormalities. a T2W sagittal image showing ill-defined intramedullary hyperintensity consistent with myelomalacia centered over L1-3 (indicated by the white lines). b $\mathrm{T} 2 \mathrm{~W}$ sagittal image showing more extensive hyperintensity consistent with myelomalacia. c T2W sagittal image showing a small, well-defined hyperintense region representative of focal cystic change (arrow). d T2W sagittal image showing a well-defined hyperintensity extending more than one vertebral body in length consistent with syringomyelia with inset showing sagittal HASTE image of the pictured region. e T2W sagittal image showing intramedullary hypointensity consistent with extensive intraparenchymal fibrosis (extent indicated by white lines). f T2W sagittal image of hypointensity consistent with more focal fibrosis (extent indicated by white lines)

performed within $24 \mathrm{~h}$ of presentation to the hospital in $18 / 19$ dogs; the precise timing of surgery was unavailable in $1 \mathrm{dog}$. The remaining eight dogs were all managed medically. Median duration of injury (time from injury to imaging) was 13 months (IQR: 8-28) with 18 dogs having a duration $\leq 13$ months and 17 dogs with a duration $>13$ months. Median open field score was 2 (IQR: 0-3). Two dogs were independently ambulatory (scores of 5 and 6), 4 dogs took some weight bearing steps (score of 4), and 29 were non-ambulatory (scores of $0-3$ ).

Lesion location ranged from the level of the 7 th thoracic vertebra to the 4th lumbar vertebra, with 23 dogs having
Table 3 Summary of quantitative imaging variables in chronically paralyzed dogs

\begin{tabular}{lll}
\hline Feature & Median & Interquartile range \\
\hline LL & $4.9 \mathrm{~cm}$ & $3.84-7.7 \mathrm{~cm}$ \\
LL:L2 & 3.5 & $2.49-5.25$ \\
MSCC & $100 \%$ & $100-100 \%$ \\
Length of 100\% MSCC $(n=29)$ & $1.94 \mathrm{~cm}$ & $1.16-2.84 \mathrm{~cm}$ \\
Length of 100\% MSCC:L2 & 1.29 & $0.72-2.16$ \\
\hline
\end{tabular}

$L L$ lesion length, $L 2$ second lumbar vertebral body length, $M S C C$ maximum spinal cord compromise

lesions at T13-L1 or cranial, 12 dogs with lesions at L1-L2 or caudal. The spinal cord was abnormal in the region of prior injury in all dogs. No dogs had extended spinal cord atrophy, while all dogs had areas within the lesion consistent with myelomalacia (Fig. 1). A lesion consistent with myelomalacia was the only abnormality noted in four dogs $(4 / 35,11 \%)$. Twenty-nine of $35 \mathrm{dogs}(83 \%)$ had an area of fluid accumulation as part of the lesion with focal cystic change present in 20/35 (57\%) and syringomyelia noted less commonly in 9/35 (26\%) (Fig. 1c, d). Among dogs with a syrinx, length ranged from 2 to 5 vertebral bodies. Findings consistent with intramedullary fibrosis within the lesion were uncommon $(6 / 35,17 \%)$, present only in dogs that had undergone surgical decompression for intervertebral disk herniation or had suffered a fracture/luxation, and, in the eight dogs in which gradient echo sequences (T2*) were performed, none had evidence of hemorrhage (Fig. 1e, f). A summary of quantitative imaging abnormalities is provided in Table 3. Maximum spinal cord compromise was $100 \%$ (no normal spinal cord tissue discernible at lesion epicenter) in 29 dogs, while 6 dogs had a maximum spinal cord compromise of less than 100\% (median: $77 \%$, IQR: 73-87). Lesion length and length of the region with no normal signal intensity varied extremely widely between dogs.

There were no relationships identified between qualitative imaging features and open field score or injury duration (Fig. 2, $p>0.05$ ). Surgical vs. medical management did not affect the presence of qualitative imaging features significantly $(p>0.05)$. Lesion location (cranial or caudal group) did not have a significant impact on open field score $(p=0.0732)$. The relationship between open field score, injury duration, or treatment for intervertebral disk herniation and quantitative variables is presented in Fig. 3. The presence of less than $100 \%$ maximum spinal cord compromise (i.e., some spinal cord tissue of normal signal intensity was present) within the lesion epicenter did not affect open field score $\left(p=0.219, p_{\mathrm{a}}=0.219\right)$. Lesion length normalized to L2 was not associated with open field score ( $\left.p=0.106, p_{\mathrm{a}}=0.212, r^{2}=0.1\right)$, but the length of the 

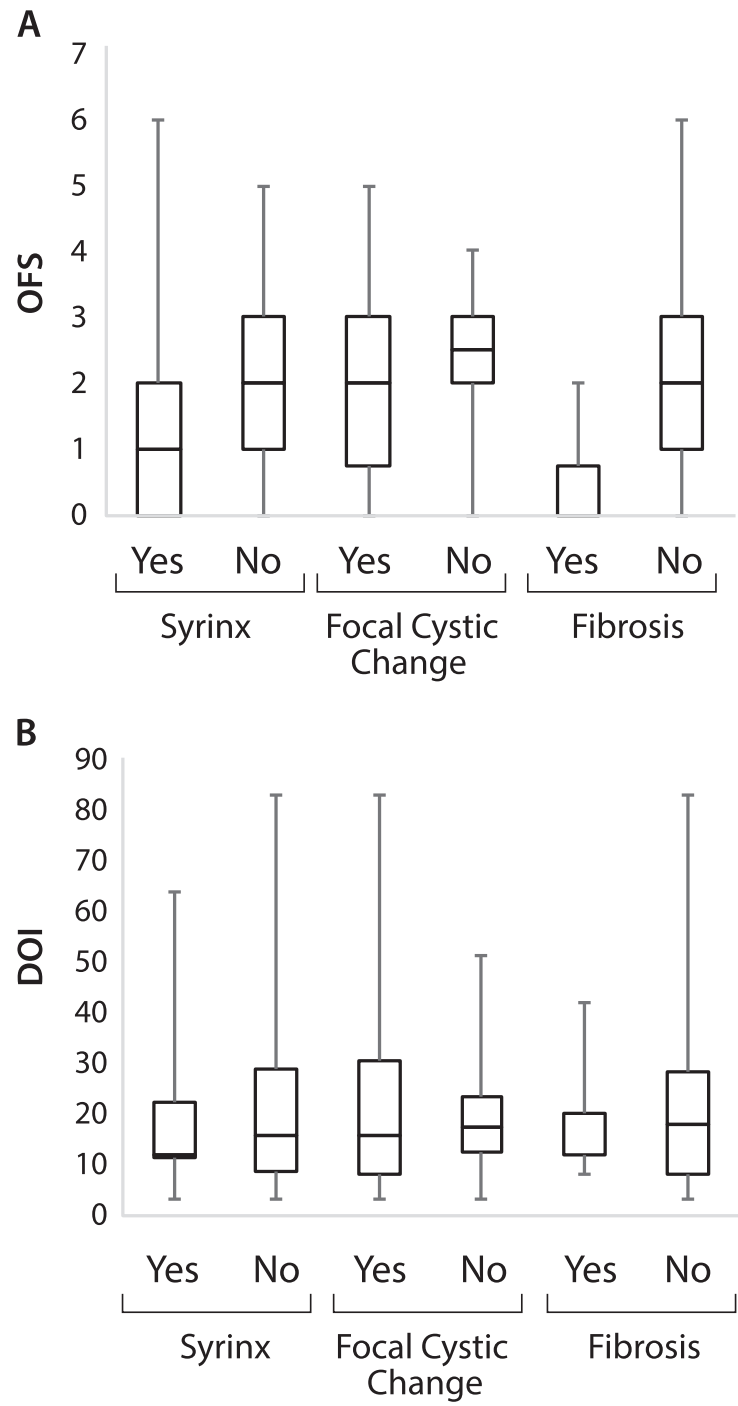

Fig. 2 Relationship between qualitative imaging features and a OFS or b DOI. No significant associations were identified $(p>0.05)$

region with $100 \%$ spinal cord compromise normalized to L2 did impact open field score $\left(p=0.0041, p_{\mathrm{a}}=0.012\right.$, $r^{2}=0.22$ ). No relationships were identified between quantitative variables and duration of injury or treatment type (medical vs. surgical) for disk herniation dogs $(p>0.05)$.

\section{Discussion}

The results of this study establish MRI lesion characteristics in a population of dogs with incomplete recovery after severe, acute SCI. Lesion appearance and extent were varied although certain features were common. Frequency of cystic change and syringomyelia were comparable to findings in humans. We demonstrated that the length of the region within the spinal cord lesion that had $100 \%$ abnormal signal intensity in the transverse plane was associated with hind limb motor function. This suggests that the craniocaudal length of apparently complete transverse myelopathy on MRI impacts the functional status in dogs judged clinically to have a complete SCI.

\section{Quantitative MRI findings}

Quantitative variables on MRI have been described for dogs with acute SCI and humans with acute and chronic SCI $[2,3,5,6,8-12,14,15,17]$. However, quantitative MRI analysis of lesions in dogs with chronic deficits after acute, complete injury is lacking. There is only one recent study that includes a small number of chronically impaired dogs, but the length of the lesion on $\mathrm{T} 2 \mathrm{~W}$ images was the only conventional MRI measurement examined and its relationship to function was not evaluated [21]. We utilized measurements defined for dogs with acute SCI to quantify lesion dimensions in a population of chronically impaired dogs $[2,3,12]$.

Lesion length varied widely between dogs even after adjusting by the length of L2 to account for dogs of differing body size, illustrating the continuum of injury severity in this clinically similar population. The presence and dimensions of the lesion (T2W intramedullary hyperintensity) have been associated with outcome in dogs with acute SCI, but lesion length was not associated with functional status in our population $[3,6,10,13,18]$. Additionally, $83 \%$ of dogs in this study had an area of the spinal cord with no normal signal intensity and having less than $100 \%$ maximum spinal cord compromise at the epicenter was not associated with improved motor function, suggesting that the percentage of abnormal spinal cord signal intensity on cross-section by itself does not appear to be a useful parameter in the chronic setting in this population. In an attempt to better capture injury severity, we measured the length of the spinal cord that had $100 \%$ altered signal intensity. While also variable, the effect of the length of this region (raw and adjusted by L2) on gait scores was detectable, even in our small sample size. This suggests that the length of apparent complete disruption of the spinal cord is important functionally. Conventional MRI cannot detect single or small numbers of intact axons traversing a site of injury, but our findings suggest an increased likelihood of the presence of such trans-lesional fibers as the longitudinal extent with no discernible normal tissue decreases. The potential impact of injury location must also be considered when interpreting this association. The lumbar segments contain the neuronal network known as the CPG, which has an important role in gait generation [35-37]. While it is possible dogs with lesions disrupting the lumbar segments fare differently than those with lesions located more 

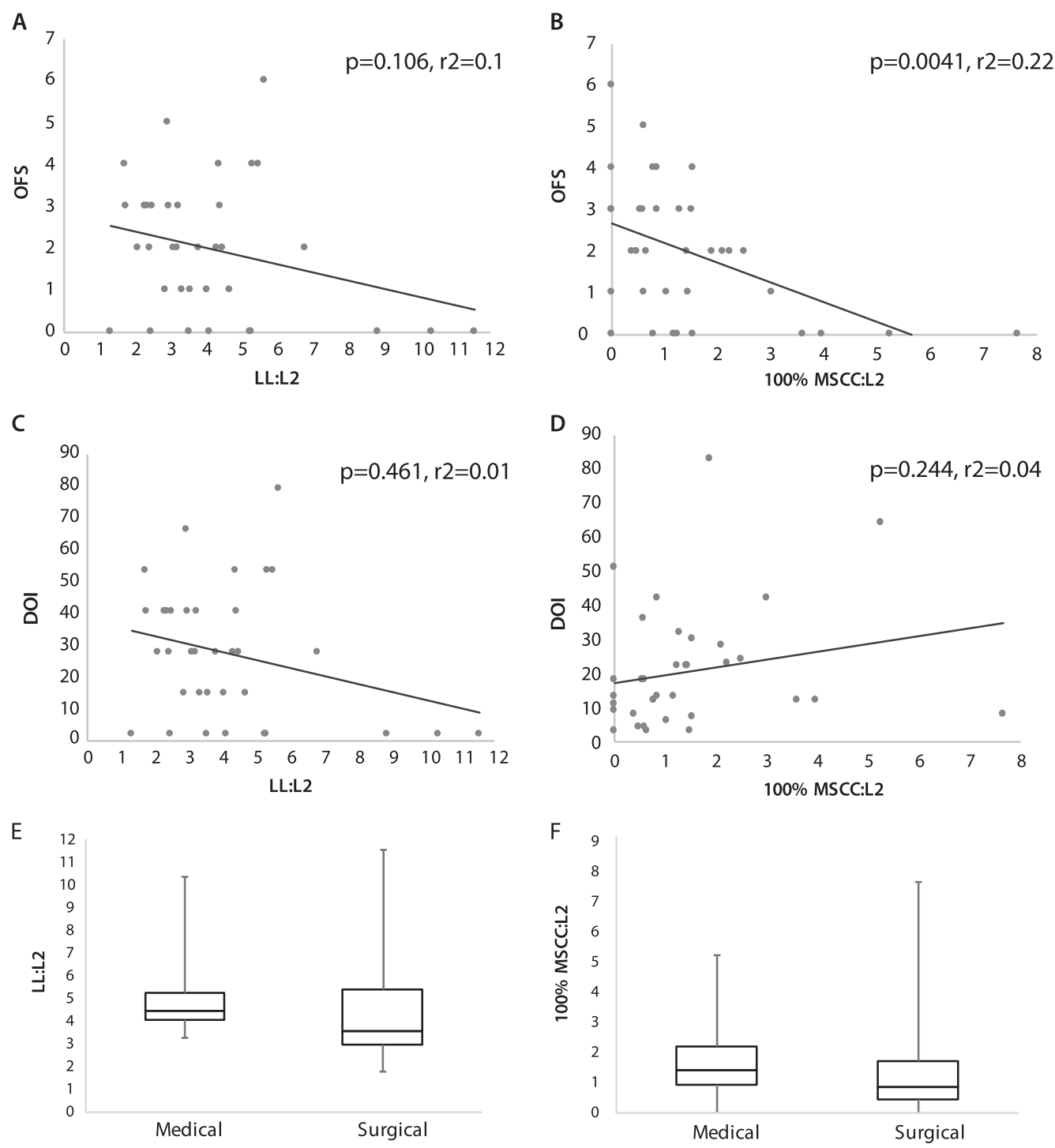

Fig. 3 Relationship between quantitative imaging abnormalities and motor function, injury duration, or treatment type. a, b OFS and LL:L2 $\left(p=0.106, r^{2}=0.1\right)$ or $100 \%$ MSCC:L2 $\left(p=00041, r^{2}=0.22\right)$; c, d DOI and LL:L2 $\left(p=0.461, r^{2}=0.01\right)$ or $100 \%$ MSCC:L2 $(p=0.244$, $\left.r^{2}=0.04\right)$; e, f treatment type (medical or surgical) for dogs with IVDH and LL:L2 $(p=0.265)$ or 100\% MSCC $(p=0.455)$. OFS open field score, $L L$ lesion length $(\mathrm{cm}), 100 \%$ MSCC length of $100 \%$ compromised spinal cord region $(\mathrm{cm}), D O I$ duration of injury, $I V D H$ intervertebral disk herniation

cranially along the thoracolumbar spinal cord, we were unable to detect an effect.

\section{Qualitative MRI features: myelomalacia, cystic change, and syringomyelia}

Qualitative features on MRI have been reported in dogs with acute SCI, but there are no studies describing the frequency of major pathologic features in dogs with chronic SCI $[2,5,6,9-11,14]$. We adapted our definitions from reports in humans in which qualitative MRI characteristics of chronic SCI include intramedullary changes such as myelomalacia, syrinx or cyst formation, as well as spinal cord compression, atrophy, disruption, and tethering $[22,24,25,27]$.

While the spinal cord can appear normal on MRI in dogs that recover clinically from acute SCI, all dogs in our study had persistent lesions with areas consistent with myelomalacia, supporting the presence of permanent damage to the spinal cord after severe injury [19]. Malacia is also common in humans with chronic SCI and can be differentiated from fluid-filled cavitation (syrinx or cyst formation) based on imaging characteristics, although these abnormalities can occur concurrently [25, 27]. Syringomyelia was identified in approximately one quarter of dogs, while focal cystic change was present in just over half the dogs. This is 
comparable to humans in which approximately $21-28 \%$ of chronic SCI patients develop post-traumatic syringomyelia and $30-50 \%$ demonstrate some degree of cystic change [38-40]. The precise cause of post-traumatic syringomyelia in people is not well understood and it remains unclear if focal fluid pocketing secondary to areas of myelomalacia is a necessary precursor to development of this condition [38]. In a rodent model of SCI, concurrent induction of arachnoiditis (to potentiate syrinx development) resulted in larger syrinxes and greater locomotor dysfunction at 6-weeks post-injury compared to rats with SCI alone, suggesting that development of post-traumatic syringomyelia contributes to injury severity and influences recovery of motor function [41]. While only a small percentage of people develop symptomatic syrinxes, affected patients experience neurologic deterioration after a period of static signs and effective treatment is challenging [38, 39, 42, 43]. No dogs in our study demonstrated an overt deterioration in neurologic status after reaching a plateau following injury and the importance of identifying a syrinx on MRI in dogs with chronic SCI is unknown. The presence of a syrinx or focal cystic change did not affect functional status and we did not identify a relationship between their presence and injury duration. Serial imaging in the chronic setting would be needed to determine if dogs with focal cystic change go on to develop overt syringomyelia over time or if there is an association between progressive syrinx elongation and outcome in dogs. Given the similar frequency of development of this abnormality between dogs and people, dogs with spontaneous SCI might be a useful population in which to continue to investigate causes and interventions for post-traumatic syringomyelia.

\section{Qualitative MRI features: intramedullary fibrosis}

Findings consistent with intramedullary fibrosis were relatively uncommon, occurring in about one-third of cases. While fibrosis was not associated with functional status or injury duration, only dogs that suffered disk herniation and underwent surgery or had vertebral column trauma had findings consistent with fibrosis. Spinal fractures and luxations in people secondary to trauma can result in dural lacerations and predispose to the development of fibrous scarring, which might explain the presence of this abnormality in our cases of trauma [44-46]. Additionally, some veterinary neurosurgeons perform a durotomy following decompression of the spinal cord in dogs with acute, severe intervertebral disk herniation with the intended goal of relieving intramedullary pressure and improving spinal cord blood flow [47, 48]. The dog illustrated in Fig. 1e, for example, had undergone a multilevel laminectomy and durotomy. It has been shown in experimental SCI in rodents that dural disruption results in invasion of fibroblasts into the spinal cord parenchyma and that repair of such damage (via duroplasty) reduces fibrous scarring $[49,50]$. While determination of the status of the dura at the time of injury was not possible in this study, our findings might reflect a similar response to damage to the dura in dogs with SCI.

\section{Qualitative MRI features: atrophy}

We did not identify extended atrophy associated with the lesion in any dogs. This is distinct from human SCI patients in which extended atrophy was the most common imaging finding in those imaged more than 20 years after injury [27]. However, this finding was most commonly associated with cervical injuries, the marked variability in spinal cord diameter seen across dog breeds is not encountered, and precise definitions of atrophy for both the cervical and thoracic cord have been established [27]. The presence of atrophy in our population might have been difficult to discern objectively given the variable length and location of lesions and differing thoracolumbar spinal cord diameter among dogs of different breeds and body sizes. Since approximately half of the dogs had an injury duration of about a year or less, it is also possible that atrophy could become apparent with greater chronicity.

\section{Study limitations}

Limitations of this study include the relatively small sample size with variable cause and treatment of the SCI, the inherent subjectivity of imaging-based definitions, and lack of histopathologic confirmation since this is a pet dog population. Additionally, univariate analysis was used to determine associations and, as such, significant results should be interpreted with caution. Further investigation is indicated in a larger number of dogs using multivariate analysis to account for potential confounding factors. While myelomalacia was the most likely explanation for illdefined areas of $\mathrm{T} 2$ hyperintensity within the spinal cord under chronic conditions, other causes such as edema or inflammation could not be excluded. While all lesions were easy to identify, it is also possible that truncation artifact mimicked the intramedullary hyperintensity obscuring the true cranial and caudal extent of the lesion and impacted lesion length measurements [51]. Given the chronicity of these injuries, fibrosis was most likely for areas of $\mathrm{T} 1$ and T2 hypointensity identified within the lesion but incidental mineralization or even hemorrhage cannot be excluded. Gradient echo sequences were only performed in a small number of dogs $(n=8)$ hindering the ability to rule out hemorrhage. However, five of the eight dogs were imaged within 9 months of injury and none showed changes consistent with hemorrhage, suggesting the likelihood of such a finding would be even less with increasing chronicity. As 
mentioned above, extended atrophy as a feature of the lesion was not identified but the possibility of its presence was not excluded. Measuring atrophy distant from the site of injury might avoid the complexities of the lesion itself while still providing a means to capture injury severity.

\section{Conclusions}

We have described the spectrum of MRI changes in a population of chronically paralyzed dogs and demonstrated that the length of the spinal cord region with $100 \%$ abnormal signal intensity in the transverse plane is associated with motor function. This population of dogs shows comparable MRI changes to people with chronic SCI and, like people, dogs with apparently clinically complete injuries show a spectrum of injury severity. Our findings support the use of dogs with naturally occurring, severe SCI as a model of chronic paralysis in people.

\section{Data archiving}

All relevant data are within this manuscript and raw data are archived by the authors.

Acknowledgements T32 OD011130-Comparative Medicine and Translational Research Training Program and the Morris Animal Foundation grant 10CA-04O.

\section{Compliance with ethical standards}

Conflict of interest The authors declare that they have no competing interests.

\section{References}

1. Cooper JJ, Young BD, Griffin JF, Fosgate GT, Levine JM. Comparison between noncontrast computed tomography and magnetic resonance imaging for detection and characterization of thoracolumbar myelopathy caused by intervertebral disk herniation in dogs. Vet Radiol Ultrasound. 2014;55:182-9.

2. De Risio L, Adams V, Dennis R, McConnell F, Platt S. Magnetic resonance imaging findings and clinical associations in 52 dogs with suspected ischemic myelopathy. J Vet Intern Med. 2007;21:1290-8.

3. De Risio L, Adams V, Dennis R, McConnell FJ. Association of clinical and magnetic resonance imaging findings with outcome in dogs with presumptive acute noncompressive nucleus pulposus extrusion: 42 cases (2000-2007). J Am Vet Med Assoc. 2009;234:495-504.

4. Granger N, Carwardine D. Acute spinal cord injury tetraplegia and paraplegia in small animals. Vet Clin Small Anim. 2014;44:1131-56.

5. Johnson P, Beltran E, Dennis R, Taeymans O. Magnetic resonance imaging characteristics of suspected vertebral instability associated with fracture or subluxation in eleven dogs. Vet Radiol Ultrasound. 2012;53:552-9.

6. Levine JM, Fosgate GT, Chen AV, Rushing R, Nghiem PP, Platt $\mathrm{SR}$, et al. Magnetic resonance imaging in dogs with neurologic impairment due to acute thoracic and lumbar intervertebral disk herniation. J Vet Intern Med. 2009;23:1220-6.
7. Park EH, White GA, Tieber LM. Mechanisms of injury and emergency care of acute spinal cord injury in dogs and cats. J Vet Emerg Crit Care. 2012;22:160-78.

8. Beltran E, Dennis R, Doyle V, De Stefani A, Holloway A, De Risio L. Clinical and magnetic resonance imaging features of canine compressive cervical myelopathy with suspected hydrated nucleus pulposus extrusion. J Small Anim Pract. 2012;53:101-7.

9. Besalti O, Pekcan Z, Sirin YS, Erbas G. Magnetic resonance imaging findings in dogs with thoracolumbar intervertebral disk disease: 69 cases (1997-2005). J Am Vet Med Assoc. 2006;228:902-8.

10. Boekhoff TM, Flieshardt C, Ensinger EM, Fork M, Kramer S, Tipold A. Quantitative magnetic resonance imaging characteristics: evaluation of prognostic value in the dog as a translational model for spinal cord injury. J Spinal Disord Tech. 2012;25:E81-7.

11. Chang Y, Dennis R, Platt SR, Penderis J. Magnetic resonance imaging of traumatic intervertebral disc extension in dogs. Vet Rec. 2007;160:795-99.

12. De Risio L, Adams V, Dennis R, McConnel FJ, Platt SR. Association of clinical and magnetic resonance imaging findings with outcome in dogs suspected to have ischemic myelopathy: 50 cases (2000-2006). J Am Vet Med Assoc. 2008;233:129-35.

13. Griffin JF, Davis MC, Cohen ND, Young BD, Levine JM. Quantitative magnetic resonance imaging in a naturally occurring canine model of spinal cord injury. Spinal Cord. 2015;53:278-84.

14. Henke D, Gorgas D, Flegel T, Vandevelde M, Lang J, Doherr MG, et al. Magnetic resonance imaging findings in dogs with traumatic intervertebral disk extrusion with or without spinal cord compression: 31 cases (2006-2010). J Am Vet Med Assoc. 2013;242:217-22.

15. Nakamoto Y, Ozawa T, Katakabe K, Nishiya K, Yasuda N, Mashita T, et al. Fibrocartilaginous embolism of the spinal cord diagnosed by characteristic clinical findings and magnetic resonance imaging in 26 dogs. J Vet Med Sci. 2009;71:171-76.

16. Penning V, Platt SR, Dennis R, Cappello R, Adams V. Association of spinal cord compression seen on magnetic resonance imaging with clinical outcome in 67 dogs with thoracolumbar intervertebral disc extrusion. J Small Anim Pract. 2006;47:644-50.

17. Wang-Leandro A, Siedenburg JS, Hobert MK, Dziallas P, Rohn K, Stein VM, et al. Comparison of preoperative quantitative magnetic resonance imaging and clinical assessment of deep pain perception as prognostic tools for early recovery of motor function in paraplegic dogs with intervertebral disk herniations. J Vet Intern Med. 2017;31:842-48.

18. Ito $\mathrm{D}$, Matsunaga $\mathrm{S}$, Jeffery $\mathrm{ND}$, Sasaki $\mathrm{N}$, Nishimura $\mathrm{R}$, Mochizuki $\mathrm{M}$, et al. Prognostic value of magnetic resonance imaging in dogs with paraplegia caused by thoracolumbar intervertebral disk extrusion: 77 cases (2000-2003). J Am Vet Med Assoc. 2005;227:1454-60.

19. Forterre F, Gorgas D, Dickomeit M, Jaggy A, Lang J, Spreng D. Incidence of spinal compressive lesions in chondrodystrophic dogs with abnormal recovery after hemilaminectomy for treatment of thoracolumbar disc disease: a prospective magnetic resonance imaging study. Vet Surg. 2010;39:165-72.

20. Hu R, Zhou J, Luo C, Lin J, Wang X, Li X, et al. Glial scar and neuroregeneration: histological, functional, and magnetic resonance imaging analysis in chronic spinal cord injury. J Neurosurg Spine. 2010;13:169-80.

21. Wang-Leandro A, Hobert MK, Alisauskaite N, Dziallas P, Rohn K, Stein VM et al. Spontaneous acute and chronic spinal cord injuries in paraplegic dogs: a comparative study of in vivo diffusion tensor imaging. Spinal Cord. 2017; 1-9: doi:10.1038/sc.2017.83

22. Curati WL, Kingsley DPE, Kendall BE, Moseley IF. MRI in chronic spinal cord trauma. Neuroradiology. 1992;35:30-5. 
23. Miyanji F, Furlan JC, Aarabi B, Arnold PM, Fehlings MG. Acute cervical traumatic spinal cord injury: MR imaging findings correlated with neurologic outcome-prospective study with 100 consecutive patients. Radiology. 2007;243:820-27.

24. Potter K, Saifuddin A. Pictoral review MRI of chronic spinal cord injury. Br J Radiol. 2003;76:347-52.

25. Quencer RM, Sheldon JJ, Post MJ, Diaz RD, Montalvo BM, Green BA, et al. MRI of the chronically injured cervical spinal cord. Am J Roentgenol. 1986;147:125-32.

26. Taneichi H, Abumi K, Kaneda K, Terae S. Monitoring the evolution of intramedullary lesions in cervical spinal cord injury. Qualitative and quantitative analysis with sequential MR imaging. Paraplegia. 1994;32:9-18.

27. Wang D, Bodley R, Sett P, Gardner B, Frankel H. A clinical magnetic resonance imaging study of the traumatized spinal cord more than 20 years following injury. Paraplegia. 1996;34:65-81.

28. Freund P, Weiskopf N, Ashburner J, Wolf K, Sutter R, Altmann $\mathrm{DR}$, et al. MRI investigation of the sensorimotor cortex and the corticospinal tract after acute spinal cord injury: a prospective longitudinal study. Lancet Neurol. 2013;12:873-81.

29. Hou J, Xiang Z, Yan R, Zhao M, Wu Y, Zhong J, et al. Motor recovery at 6 months after admission is related to structural and functional reorganization of the spine and brain in patients with spinal cord injury. Hum Brain Mapp. 2016;37:2195-09.

30. Lundell H, Barthelemy D, Skimminge A, Dyrby TB, Biering-Sorenson F, Nielson JB. Independent spinal cord atrophy measures correlate to motor and sensory deficits in individuals with spinal cord injury. Spinal Cord. 2011;49:70-5.

31. Moore SA, Granger N, Olby NJ, Spitzbarth I, Jeffery ND, Tipold A, et al. Targeting translational successes through CANSORT-SCI: using pet dogs to identify effective treatments for spinal cord injury. J Neurotrauma. 2017; doi:10.1089/neu. 2016.4745.

32. Olby NJ, De Risio L, Muñana KR, Wosar MA, Skeen TM, Sharp NJ, et al. Development of a functional scoring system in dogs with acute spinal cord injuries. Am J Vet Res. 2001;62:1624-28.

33. Olby NJ, Muguet-Chanoit AC, Lim JH, Davidian M, Mariani CL, Freeman AC, et al. A placebo-controlled, prospective randomized clinical trial of polyethylene glycol and methylprednisolone sodium succinate in dogs with intervertebral disk herniation. J Vet Intern Med. 2016;30:206-14.

34. Seiler GS, Robertson ID, Mai W, Widmer WR, Suran J, Nemanic $\mathrm{S}$, et al. Usefulness of a half-Fourier acquisition single-shot turbo spin-echo pulse sequence in identifying arachnoid diverticula in dogs. Vet Radiol Ultrasound. 2012;53:157-61.

35. Gerasimenko YP, Makarovskii AN, Nikitin OA. Control of locomotor activity in humans and animals in the absence of supraspinal influences. Neurosci Behav Physiol. 2002;32:417-23.
36. Guertin PA. The mammalian central pattern generator for locomotion. Brain Res Rev. 2009;62:45-56.

37. Rossignol S, Bouyer L, Barthelemy D, Langlet C, Leblond H. Recovery of locomotion in the cat following spinal cord lesions. Brain Res Rev. 2002;40:257-66.

38. Brodbelt AR, Stoodley MA. Post-traumatic syringomyelia: a review. J Clin Neurosci. 2003;10:401-8.

39. Perrouin-Verbe B, Lenne-Aurier K, Robert R, Auffray-Calvier E, Richard I, Mauduyt de la Greve I. Post-traumatic syringomyelia and post-traumatic spinal canal stenosis: a direct relationship: review of 75 patients with a spinal cord injury. Spinal Cord. 1998;36:137-43.

40. Austin JW, Afshar M, Fehlings MG. The relationship between localized subarachnoid inflammation and parenchymal pathophysiology after spinal cord injury. $J$ Neurotrauma. 2012;29:1838-49.

41. Seki T, Fehlings MG. Mechanistic insights into posttraumatic syringomyelia based on a novel in vivo animal model. J Neurosurg Spine. 2008;8:365-75.

42. Karam Y, Hitchon PW, Mhanna NE, He W, Noeller J. Post-traumatic syringomyelia: outcome predictors. Clin Neurol Neurosurg. 2014;124:44-50.

43. Krebs J, Koch HG, Hartmann K, Frotzler A. The characteristics of posttraumatic syringomyelia. 2015; doi:10.1038/sc.2015.218.

44. Keenen TL, Antony J, Benson DR. Dural tears associated with lumbar burst fractures. J Orthop Trauma. 1990;4:243-45.

45. Pau A, Silverstro C, Carta F. Can lacerations of the thoracolumbar dura be predicted on the basis of radiological patterns of the spinal fractures. Acta Neurochir. 1994;129:186-87.

46. Pickett J, Blumenkopf B. Dural lacerations and thoracolumbar fractures. J Spinal Disord. 1989;2:99-103.

47. Blaser A, Lang J, Henke D, Doherr MG, Adami C, Forterre F. Influence of durotomy on laser-doppler measurement of spinal cord blood flow in chondrodystrophic dogs with thoracolumbar disk extrusion. Vet Surg. 2012;41:221-27.

48. Loughin CA, Dewey CW, Ringwood PB, Pettigrew RW, Ken M, Budsberg SC. Effect of durotomy on functional outcome of dogs with type I thoracolumbar disc extrusion and absent deep pain perception. Vet Comp Orthop Traumatol. 2005;18:141-46.

49. Fernandez E, Pallini R. Connective tissue scarring in experimental spinal cord lesions: significance of dural continuity and role of epidural tissues. Acta Neurochir. 1985;76:145-48.

50. Iannotti C, Zhang YP, Shields L, Han Y, Burke DA, Xu X, et al. Dural repair reduces connective tissue scar invasion and cystic cavity formation after acute spinal cord laceration injury in adult rats. J Neurotrauma. 2006;23:853-65.

51. Gregori T, Lam R, Priestnall SL, Lamb CR. Truncation artifact in magnetic resonance images of the canine spinal cord. Vet Radiol Ultrasound. 2016;57:582-86. 